\title{
Effects of Negative Emotional Experience and Behavior Impulsivity on Non-suicidal Self-injury in Adolescents: A Follow-up Study
}

\section{YOU Jia-ning*, DENG Bao-ping, ZHANG Ming-qin and LI Sheng-teng}

Center for Studies of Psychological Applican \& School of Psychology, South China Normal University, China

\begin{abstract}
Objective: To explore the predictive utilities of negative emotional experiences and behavior impulsivity on nonsuicidal self-injury ( $\mathrm{NSSI}$ ) in adolescents, and to test the moderating effects of behavior impulsivity on the relation between negative emotional experiences and NSSI.
\end{abstract}

Methods: A total of 3600 middle school students completed questionnaires three times over a 1.5 year period, six months apart. At the first time, we measured the occurrence of NSSI, dissociative experience, depression and behavior impulsivity. At the second and third times, we measured the occurrence of NSSI. We used generalized estimating equations to analyze the longitudinal data.

Results: Baseline levels of depression, dissociative experience and behavior impulsivity significantly predicted subsequent NSSI; but the interaction effects of behavior impulsivity and depression, dissociative experience on NSSI was not significant.

Conclusion: This study supports the emotion regulation function of NSSI. Behavior impulsivity also significantly influences future engagement in this behavior.

Keywords: Adolescents; Non-suicidal self-injury; Negative emotions; Behavior impulsivity

\section{Introduction}

Non-suicidal Self-injury (NSSI) refers to the direct, deliberate, and socially unacceptable destruction of one's body tissue without conscious suicidal intent [1]. Common NSSI acts include self-cutting, burning, biting, scratching, and self-hitting. Previous research showed that the lifetime prevalence of NSSI ranged between $15 \%$ and $30.7 \%$, the 12 -month prevalence ranged from $7.5 \%$ to $22 \%$, and the 6 -month prevalence ranged from $13.9 \%$ to $16.3 \%$ [2]. As a highly dangerous behavior, NSSI can predict future suicide attempt [3]. Thus, it is important to understand why adolescents engage in NSSI.

In view of the prevalence of adolescent NSSI and its severe consequences, researchers start to probe into the reasons or functions of NSSI. Some researchers believe that NSSI primarily serves the emotion regulation function [4,5]. Specifically, this function means that when an individual is experiencing intensely negative emotions, he or she is likely to engage in NSSI to reduce or relieve these unwanted emotions $[1,6]$. However, the emotion regulation function still could not fully explain why people specifically choose NSSI rather than other less destructive and more appropriate behaviors. To partly address this question, Nock proposed a "Pragmatic Hypothesis" in 2009. According to this hypothesis, NSSI is the most convenient and efficient coping behavior as compared to talking to others, drinking, and exercise. NSSI could be conducted at any place, at any time and it does not need the presence of other people. Therefore, highly impulsive individuals are more likely to engage in NSSI when they are suffering intense negative emotions [7]. Based on this hypothesis, behavioral impulsivity may act as a moderator of the relation between negative emotional experience and NSSI.

With the aim of testing this hypothesis, we conducted a follow-up study with secondary school students. We used Generalized Estimating Equation (GEE) to examine the effects of negative emotional experiences (depression and dissociative experience) and behavioral impulsivity on later engagement in NSSI, as well as the moderating effect and behavioral impulsivity. GEE is suitable for longitudinal data analysis, and can help to explore the impact of independent variables on subsequent changes of dependent variables.

\section{Participants and Methods}

\section{Participants}

Participants were students from eight secondary schools. There were data losses in each time of the testing due to the students' in and out every year in every class. At the baseline assessment, 5423 students (52.7\% girls) participated. They aged between 12 and 18 years $(\mathrm{M}=14.63, \mathrm{SD}=1.25)$. At the second assessment, 6911 students $(52.6 \%$ girls) participated and 3999 of them were successfully followed from the Wave 1 sample. At the third assessment, 6831 students (52.6\% girls) participated and 3600 of them were successfully followed all three times. The follow-up sample and the attrited sample had no significant difference on demographic and all studied variables.

\section{Measurements}

NSSI behavior: We measured seven NSSI behaviors in this study. They were: self-cutting, burning, biting, punching, scratching, inserting sharp objects into nail or skin, and banging the head or other parts of the body toward the wall. At the first assessment, participants were asked "In the past 12 months, have you deliberately harmed yourself (without suicidal intent)?" At the second and third assessment, the time frame was changed to the past six months. Participants rated each item on a 4-point scale, ranging from 1 "Never", 2 "1 to 2 times", 3 " 3 to 5 times", to 4 " 6 times or above". The summation of the scores of the seven items was used as the total frequency of NSSI. The Crobach's a coefficient in this study was 0.79 for the first assessment, 0.74 for the second assessment, and 0.79 for the third assessment.

*Corresponding author: YOU Jia-ning, Center for Studies of Psychological Applican \& School of Psychology, South China Normal University, Guangzhou 510631, China, E-mail: youjianing@gmail.com

Received June 14, 2013; Accepted July 26, 2013; Published July 30, 2013

Citation: Jia-ning Y, Bao-ping D, Ming-qin Z, Sheng-teng LI (2013) Effects of Negative Emotional Experience and Behavior Impulsivity on Non-suicidal Self-injury in Adolescents: A Follow-up Study. J Psychol Abnorm Child 2: 103 doi:10.4172/2329-9525.1000103

Copyright: ( 2013 Jia-ning Y, et al. This is an open-access article distributed under the terms of the Creative Commons Attribution License, which permits unrestricted use, distribution, and reproduction in any medium, provided the original author and source are credited. 
Behavior impulsivity: Items of this scale were selected from the impulsivity section of the Diagnostic Interview for Borderlines-Revised; DIB-R developed by Zanarini et al. [8]. 10 kinds of impulse behaviors has being measured, including binge eating, impulsive buying, falling in love at first sight, debating, fighting, injuring others, alcoholic intemperance, substance abuse, deliberate damage amd ad hominem. Participants rated each item on a 4-point scale, ranging from 1 "Never", 2 " 1 to 2 times", 3 " 3 to 5 times", to 4 " 6 times or above". The Crobach's a coefficient of this scale was 0.69 in this study.

Dissociative experiences: Three items of "Not feeling the selfexistence when excessively painful", "Feeling the self-dissociation from the real world when excessively painful", and "Self-feeling being dissociative from the self-body when excessively painful" were quoted from $D I B-R$ Zanarini et al. [8] to measure the dissociative experiences. Participants rated each item on a 4-point scale, ranging from 1 "Very disagree", 2 "disagree", 3 "agree", to 4 "very agree". The Crobach's a coefficient of this scale was 0.91 in this study.

Depression: The 7-item Depression subscale of the Depression Anxiety Stress Scale-21 items; DASS21 developed by Taouk et al. [9] was used to measure depression. Participants rated each item on a 3-point scale ranging from "not suitable at all" to "very suitable to describe me". This scale had a Crobach's $\alpha$ value of 0.86 in this study.

\section{Procedure and data analysis}

We conducted three times of assessment, six months apart. In the first assessment, participants completed questionnaires assessing NSSI, behavioral impulsivity, depression and dissociative experience. In the second and third assessment, participants were assessed on their NSSI behavior.

We conducted correlational analysis and GEE. All data analyses were conducted with SPSS 16.0.

\section{Results}

\section{Descriptive statistics}

For Wave 1, the prevalence of NSSI was $18.1 \%$, with girls $(20.6 \%$, $\mathrm{n}=410)$ being significantly more likely than boys $(14.7 \%, \mathrm{n}=245)$ to conduct NSSI, $\chi^{2}(1)=18.42, p<0.001$. For Wave 2 , the prevalence of NSSI was $12.2 \%$. Girls $(14.5 \%, \mathrm{n}=296)$ were also more likely than boys $(9 \%, \mathrm{n}=140)$ to engage in NSSI, $\chi^{2}(1)=25.42, p<0.001$. For Wave 3 , the prevalence of NSSI was $8.3 \%$, with more girls $(9.6 \%, \mathrm{n}=195)$ conducting NSSI than boys $(6.3 \%, \mathrm{n}=99), \chi^{2}(1)=12.88, p<0.001$ (Table 1 ).
Table 1 presents the means and standard deviations of all studied variables and bivariate correlation coefficients among them. Results showed that depression, dissociative experience, and behavioral impulsivity positively correlated with NSSI assessed at all three waves.

Longitudinal data analysis predicting adolescent NSSI by depression, dissociative experiences and behavior impulsivity

We predicted adolescents' subsequent engagement in NSSI by depression, dissociative experiences and behavior impulsivity assessed in the first time using GEE. We also explored the moderating effects of behavioral impulsivity and those results were shown in Table 2 .

We found that depression, dissociative experiences, and behavior impulsivity significantly predicted subsequent engagement in NSSI. Increases of 1 point in the three variables increased the likelihood of engagement in NSSI by $12 \%, 22 \%$ and $24 \%$, respectively. The interaction effects of behavioral impulsivity and depression and dissociative experience were not significant (Table 2).

\section{Discussion}

\section{The developmental trajectory of NSSI in adolescents}

This study showed that the prevalence of NSSI decreased during the three times of assessment. This is not consistent with Benjamin's (2011 study). In his research, $8 \%$ of participants reported NSSI in the baseline assessment, while $18 \%$ reported NSSI after 2.5 years. Before this study, no research examined changes of NSSI prevalence [10]. For the result of the present study that the occurrence of NSSI was decreasing, we speculate that this may be because after each assessment, we reported the name of students who engaged in NSSI to the schools. Some schools conducted psychological counseling with these students. Thus, students may be less willing to report their true situation of NSSI in the subsequent assessment. Future follow-up study of NSSI should thus not only consider the research ethics, but should also take actions to reduce the tendency of responding in an unauthentic way.

\section{Longitudinal data analysis predicting NSSI by depression, dissociative experiences and behavior impulsivity}

From Table 2, we could see that depression and dissociative experience assessed in the first time significantly predicted subsequent occurrence of NSSI. This is in line with that of Guerry and Prinstein [11]. In that research, they demonstrated that baseline depressive symptoms could predict future NSSI. This result is due to that NSSI

\begin{tabular}{|c|c|c|c|c|c|c|c|}
\hline Variables & 1 & 2 & 3 & 4 & 5 & 6 & $M \pm S D$ \\
\hline 1 Depression & 1 & & & & & & $4.01 \pm 4.07$ \\
\hline 2 Dissociation & $0.55^{\star *}$ & 1 & & & & & $5.22 \pm 2.38$ \\
\hline 3 Impulsivity & $0.42^{* *}$ & $0.36^{* *}$ & 1 & & & & $12.89 \pm 2.86$ \\
\hline $4 \mathrm{NSSI}$ t1 & $0.32^{* *}$ & $0.30^{* *}$ & $0.43^{* *}$ & 1 & & & $7.49 \pm 1.49$ \\
\hline $5 \mathrm{NSSI}$ t2 & $0.25^{\star *}$ & $0.24^{\star *}$ & $0.28^{* *}$ & $0.46^{* *}$ & 1 & & $7.30 \pm 1.09$ \\
\hline $6 \mathrm{NSSI}$ t3 & $0.18^{* *}$ & $0.19^{\star *}$ & $0.24^{* *}$ & $0.38^{* *}$ & $0.56^{\star *}$ & 1 & $7.25 \pm 1.19$ \\
\hline
\end{tabular}

Note. ${ }^{*} p<0.05,{ }^{* *} p<0.01,{ }^{* * *} p<0.001$.

Table 1: Means and standard deviations of studied variables and bivariate correlations among them.

\begin{tabular}{|c|c|c|c|}
\hline & \multicolumn{2}{|c|}{ subsequent NSSI occurrence } \\
\hline Variables & $B$ & $95 \%$ confidence interval \\
\hline Dissociation & $0.12^{* * *}$ & {$[0.08,0.15]$} & 1.12 \\
\hline Depression & $0.20^{* * *}$ & {$[0.14,0.27]$} & 1.22 \\
\hline Behavior Impulsivity & $0.21^{* * *}$ & {$[0.18,0.25]$} \\
\hline
\end{tabular}

Table 2: Summary of the generalized estimating equation results predicting subsequent occurrence of nssi by depression, dissociative experiences and behavior impulsivity. 
could reduce or relieve negative emotional experiences. The unique contribution of the present study is that we present the rate of NSSI occurrence changing with the change of depression and dissociative experiences. Specifically, the $\operatorname{Exp}(\mathrm{B})$ of depression and dissociation were 1.12 and 1.22 , respectively, indicating that increases of 1 unit depression and dissociative experiences may result in increase of subsequent NSSI occurrence by $12 \%$ and $22 \%$, respectively. This again supports the emotion regulation function of NSSI.

Furthermore, behavior impulsivity also significantly predicted subsequent NSSI. The value of $\operatorname{Exp}$ (B) was 1.24, indicating that increase of 1 unit in behavioral impulsivity my result in $24 \%$ of increase in the occurrence of NSSI. Previous research showed inconsistent findings regarding the relation of behavioral impulsivity to NSSI. Using behavioral experimental methods, studies showed that there was no significant difference in impulsivity between individuals with and without NSSI $[12,13]$. Since people may behave differently in laboratories from that in the reality, results from laboratories may not generalize to everyday life. On the other hand, studies using self-report questionnaires found significant correlations between NSSI severity, frequency and impulsivity [14-16]. Moreover, self-report studies also showed that behavioral impulsivity could significantly predict adolescents' future NSSI $[17,18]$. These are consistent with results of the present study. All these findings suggest that behavioral impulsivity has a significant impact on adolescent NSSI.

\section{The moderating effect of behavior impulsivity in the relation between negative emotion experiences and NSSI}

This study tested the "Pragmatic Hypothesis" brought forth by Janis and Nock [13], namely, behavioral impulsivity acts as a moderator in the relation between negative emotional experience and NSSI. Results did not support this hypothesis. There were few researches directly examining this hypothesis in the past. To our knowledge, the only study testing this hypothesis was conducted by Bresin et al. [19]. They conducted a diary research with 67 college students who engaged in NSSI. Researchers first measured participants' trait impulsivity. In the subsequent 14 days, participants reported their negative emotions and NSSI urge every day. Results showed that for those with high trait impulsivity, negative emotion significantly predicted NSSI, while for those with low trait impulsivity; there was no relation between negative emotion and NSSI. Our finding is inconsistent with that of Bresin et al. [19], two possible reasons may account for this inconsistency. First, Bresin et al. [19] examined the predictability of negative emotions for NSSI urge experienced in the same day; while the present study examined the effects of baseline negative emotions on NSSI conducted half year and one year later. Second, Bresin et al. [19] used college students as their sample, while we used secondary school students in the present study. Since research directly testing the "Pragmatic Hypothesis" is still scarce, to get a better understanding of the reason for NSSI, efforts should still be made to further test this hypothesis by adopting different methods.

\section{References}

1. Nock MK (2009) Why do people hurt themselves? New insights into the nature and functions of self-injury. Curr Dir Psychol Sci 18: 78-83.

2. Muehlenkamp JJ, Claes L, Havertape L, Plener PL (2012) International prevalence of adolescent non-suicidal self-injury and deliberate self-harm. Child Adolesc Psychiatry Ment Health 6: 10.

3. Prinstein MJ, Nock MK, Simon V, Aikins JW, Cheah CS, et al. (2008) Longitudinal trajectories and predictors of adolescent suicidal ideation and attempts following inpatient hospitalization. J Consult Clin Psychol 76: 92-103.

4. Nock MK, Prinstein MJ (2004) A functional approach to the assessment of selfmutilative behavior. J Consult Clin Psychol 72: 885-890.

5. Klonsky ED (2009) The functions of self-injury in young adults who cut themselves: Clarifying the evidence for affect-regulation. Psychiatry Res 166 260-268.

6. Chapman AL, Gratz KL, Brown MZ (2006) Solving the puzzle of deliberate selfharm: The experiential avoidance model. Behav Res Ther 44: 371-394.

7. Cyders MA, Smith GT (2008) Emotion-based dispositions to rash action: positive and negative urgency. Psychol Bull 134: 807-828.

8. Zanarini MC, Gunderson JG, Frankenburg FR (1989) Revised Diagnostic Interview for Borderlines: Discriminating BPD from other Axis II disorders. Journal of Personality Disorders 3: 10-18.

9. Taouk M, Lovibond PF, Laube R (2001) Psychometric properties of a Chinese version of the 21-item depression anxiety stress scales (DASS21). In: Report for New South Wales Transcultural Mental Health Centre, Cumberland Hospital, Sydney.

10. Hankin BL, Abela JR (2011) Nonsuicidal self-injury in adolescence: prospective rates and risk factors in a $2 \frac{1}{2}$ year longitudinal study. Psychiatry Res 186: 6570 .

11. Guerry JD, Prinstein MJ (2010) Longitudinal prediction of adolescent nonsuicidal self-injury: Examination of a cognitive vulnerability-stress model. J Clin Child Adolesc Psychol 39: 77-89.

12. Glenn CR, Klonsky ED (2010) A multimethod analysis of impulsivity in nonsuicidal self-injury. Personal Disord 1: 67-75.

13. Janis IB, Nock MK (2009) Are self-injurers impulsive?: Results from two behavioral laboratory studies. Psychiatry Res 169: 261-267.

14. Simeon D, Stanley B, Frances A, Mann JJ, Winchel R, et al. (1992) Selfmutilation in personality disorders: psychological and biological correlates. Am J Psychiatry 149: 221-226.

15. Claes L, Vandereycken W, Vertommen H (2001) Self-injurious behaviors in eating-disordered patients. Eat Behav 2: 263-272.

16. Herpertz S, Sass H, Favazza A (1997) Impulsivity in self-mutilative behavior psychometric and biological findings. J Psychiatr Res 31: 451-465.

17. You J, Leung $F$ (2012) The role of depressive symptoms, family invalidation and behavioral impulsivity in the occurrence and repetition of non-suicidal self-injury in Chinese adolescents: A 2-year follow-up study. J Adolesc 35: 389-395.

18. You J, Leung F, Lai CM, Fu K (2012) The associations between non-suicidal self-injury and borderline personality disorder features among Chinese adolescents. J Pers Disord 26: 226-237.

19. Bresin K, Carter DL, Gordon KH (2013) The relationship between trait impulsivity, negative affective states, and urge for nonsuicidal self-injury: A daily diary study. Psychiatry Res 205: 227-231. 